\title{
Value of Computed Tomography-Defined Visceral Fat Area as a Risk Factor for Endometrial Cancer
}

\author{
Jaehyun Cho ${ }^{1}$, Jeong Sig Kim ${ }^{1 *}$, Suyeon Park ${ }^{2}$ and Woo Young Kim ${ }^{3}$ \\ ${ }^{1}$ Department of Obstetrics and Gynecology, Soonchunhyang University College of Medicine, Seoul, Republic of Korea \\ ${ }^{2}$ Department of Biostatistics, Soonchunhyang University College of Medicine, Seoul, Republic of Korea
}

${ }^{3}$ Department of Obstetrics \& Gynecology, Kangbuk Samsung Hospital, Sungkyunkwan University School of Medicine, Seoul, Republic of Korea.

*Corresponding author: Jeong Sig Kim, Department of Obstetrics and Gynecology, Soonchunhyang University Hospital Seoul. \#59

Daesagwan-ro, Yongsan-gu, Seoul 04401, Republic of Korea

\section{ARTICLE INFO}

Received: 幽 May 18, 2020

Published: 慧 June 09, 2020

Citation: Jaehyun Cho, Jeong Sig Kim, Suyeon Park, Woo Young Kim. Value of Computed Tomography-Defined Visceral Fat Area as a Risk Factor for Endometrial Cancer. Biomed J Sci \& Tech Res 28(1)2020. BJSTR. MS.ID.004609.

Keywords: Intra-Abdominal Fat; Computed Tomography; Body Fat Distribution; Endometrial Neoplasms; Case-Control Studies
ABSTRACT

Obesity is a risk factor for endometrial cancer. Recently, visceral fat has been found to be strongly associated with obesity-related carcinogenesis, more than subcutaneous fat. In this study, we hypothesized that the visceral fat measured by computed tomography (CT) contributes to the occurrence of endometrial cancer. A retrospective chart review of patients undergoing primary surgery for endometrial cancer was conducted. The volume of visceral fat was measured by CT scans performed at the fourth lumbar level for all participants. To compare body fat distributions assessed by a direct method in 52 endometrial cancer cases with age- and BMI-matched healthy community controls. Case group showed significantly higher mean visceral fat area (VFA; $76.2 \pm 25.0$ vs. $62.2 \pm 13.9 \mathrm{~cm}^{2}, \mathrm{p}=0.007$ ). The mean total fat area (TFA; $270.3 \pm$ 99.9 vs. $238.9 \pm 53.8 \mathrm{~cm}^{2}, \mathrm{p}=0.137$ ) and subcutaneous fat area (SFA;194.2 \pm 86.5 vs. $176.7 \pm 45.8 \mathrm{~cm}^{2}, \mathrm{p}=0.315$ ), however, presents no significant differences. VFA and BMI correlation $\left(r^{2}=0.299, p<0.001\right)$ was lower than to SFA $\left(r^{2}=0.528, p<0.001\right)$ and TFA $\left(\mathrm{r}^{2}=0.584, \mathrm{p}<0.001\right)$. In receiver operator characteristic (ROC) curve, at the value of $70.8 \mathrm{~cm}^{2}$, sensitivity and specificity of the case group was $55.8 \%$ and $75 \%$, respectively. The study suggests that increased visceral fat is associated with endometrial cancer. Furthermore, VFA acts as the most independent factor to BMI, it may provide additional information to the representative risks of endometrial cancer.

\section{Introduction}

The World Health Organization defines overweight and obesity as abnormal or excessive fat accumulation that may lead to health impairments. Obesity is a serious and on the rise health problem and it has more than doubled worldwide since 1980. Obesity has been associated with metabolic syndrome, diabetes, cardiovascular diseases (heart disease, stroke, and hypertension), other chronic diseases and psychological problems; obesity is the second most common cause of death that can be prevented [1].Recent studies have shown that obesity is correlated to increased risks of several cancer types, including esophagus, thyroid, postmenopausal breast, pancreas, gall bladder, colon, rectum, endometrium, kidney, and hematological malignancy $[2,3]$. In the Occident, endometrial cancer is the most common gynecologic malignancy and the incidence of endometrial cancer has increased since the mid-2000s [4]. In recent years, the number of patients with endometrial cancer has increased due to an increase in the obesity population in South Korea [5]. Several large cohort studies have shown that endometrial cancer is one of the earliest known obesity-related cancers. One large epidemiologic study reported that every 10 unit increase in body mass index (BMI) increases the relative risk of endometrial cancer by 2.89 times [6].

Histologically, endometrial cancer can be classified into two types. Type 1 makes up the majority of endometrial cancer and is mainly associated with the endocrine system, including nutritional factors and obesity. In type 1 endometrial cancer, the mechanism that incites tumors through the hormonal change related to obesity 
is referred to as 'unopposed estrogen hypotheses. Among women with chronic anovulation in the premenopausal period, progesterone insufficiency means that there is no function to counteract the estrogen. In postmenopausal women, it is hypothesized that excess weight leads to the aromatization of estrogen in the adipose tissue which in turn causes the exposure to highly concentrated estrogen. Potischman, et al. reported that high levels of androstenedione and testosterone increase the risks of endometrial cancer, and the rise of estrone and estradiol levels also contributes to its risk [7].

When visceral adipose tissue is excessive, adipokines, including leptin and interleukin, are secreted, causing chronic inflammation

\section{Materials and Methods}

\section{Patient Recruitment}

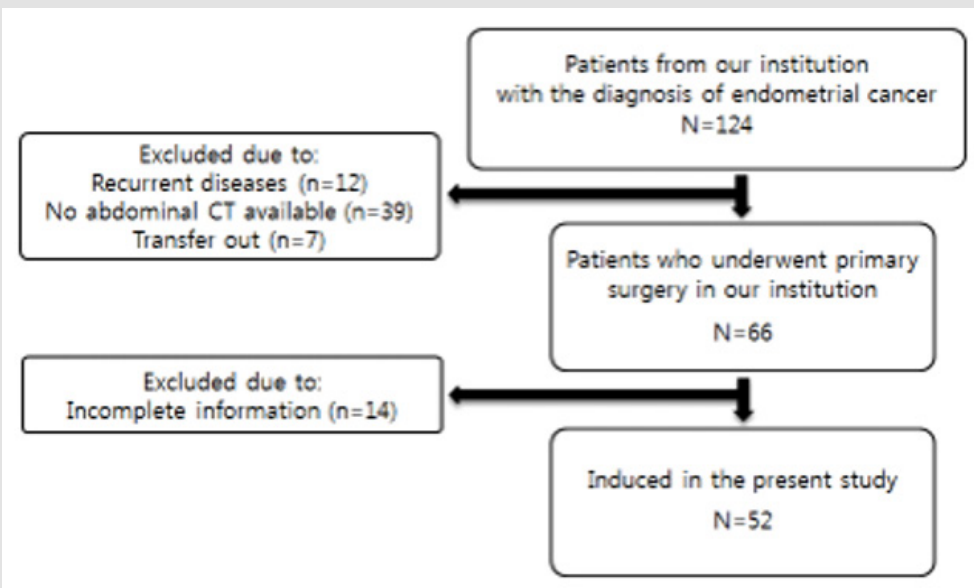

Figure 1: Flow Chart of Patient Enrollment.

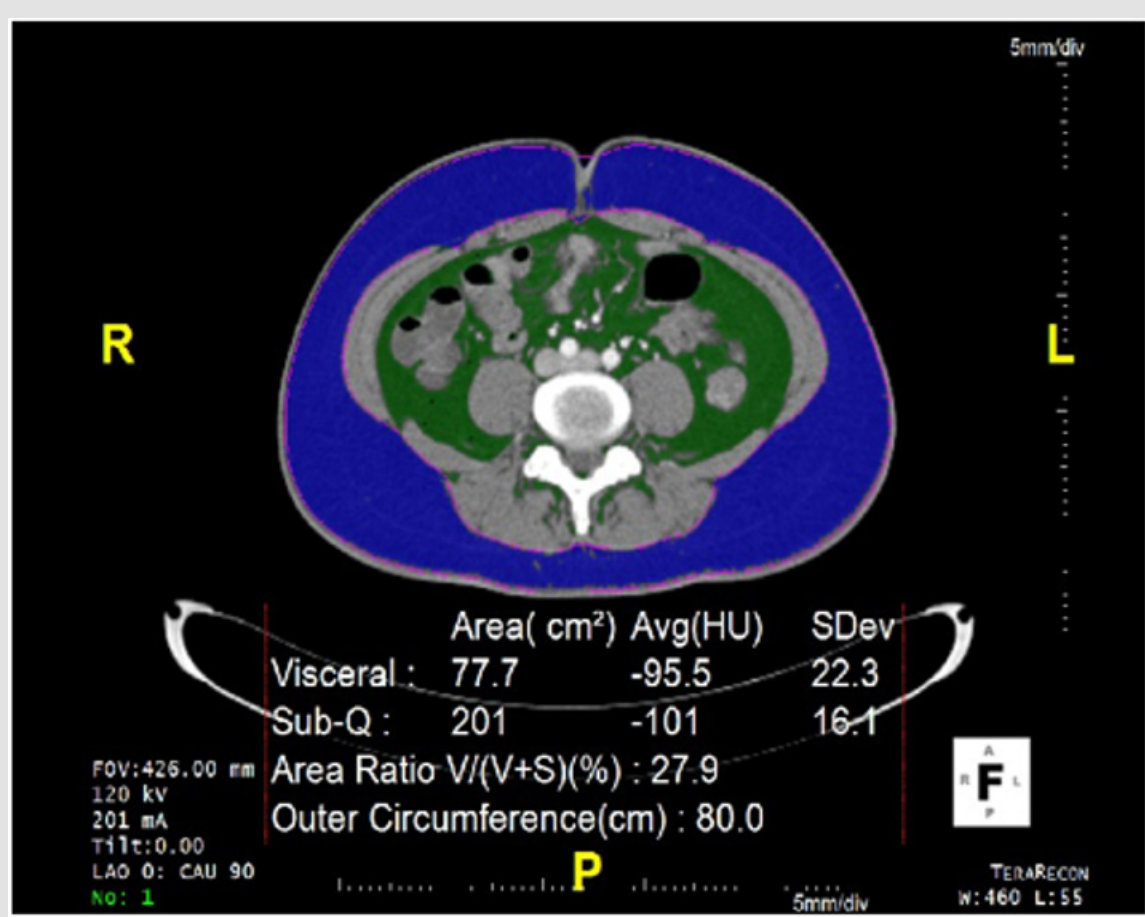

Figure 2: CT image. 
We searched our medical records database to identify all endometrial cancer patients. From 2010 to 2014, a total of 124 patients were diagnosed with endometrial cancer with biopsy and a total of 52 final case groups were selected. Figure 1 shows the flow chart of the patient enrollment process throughout the study. Patient data, including age, BMI, histological subtype, was collected from a retrospective database. The control group included 854 patients who did not have evidence of endometrial cancer with CT scan, at the same institution's health promotion center for the same period. To balance case and control groups, the propensity score (PS) method was performed by using Matching packages ( $\mathrm{R}$ version 3.1.2) [9]. PSs were calculated using a logistic regression model and the following covariates: age and BMI. Using PSs, control subject was individually matched to endometrial cancer patients and a total of 104 subjects generated with 52 for control group and case group respectively (Figure 2-4).

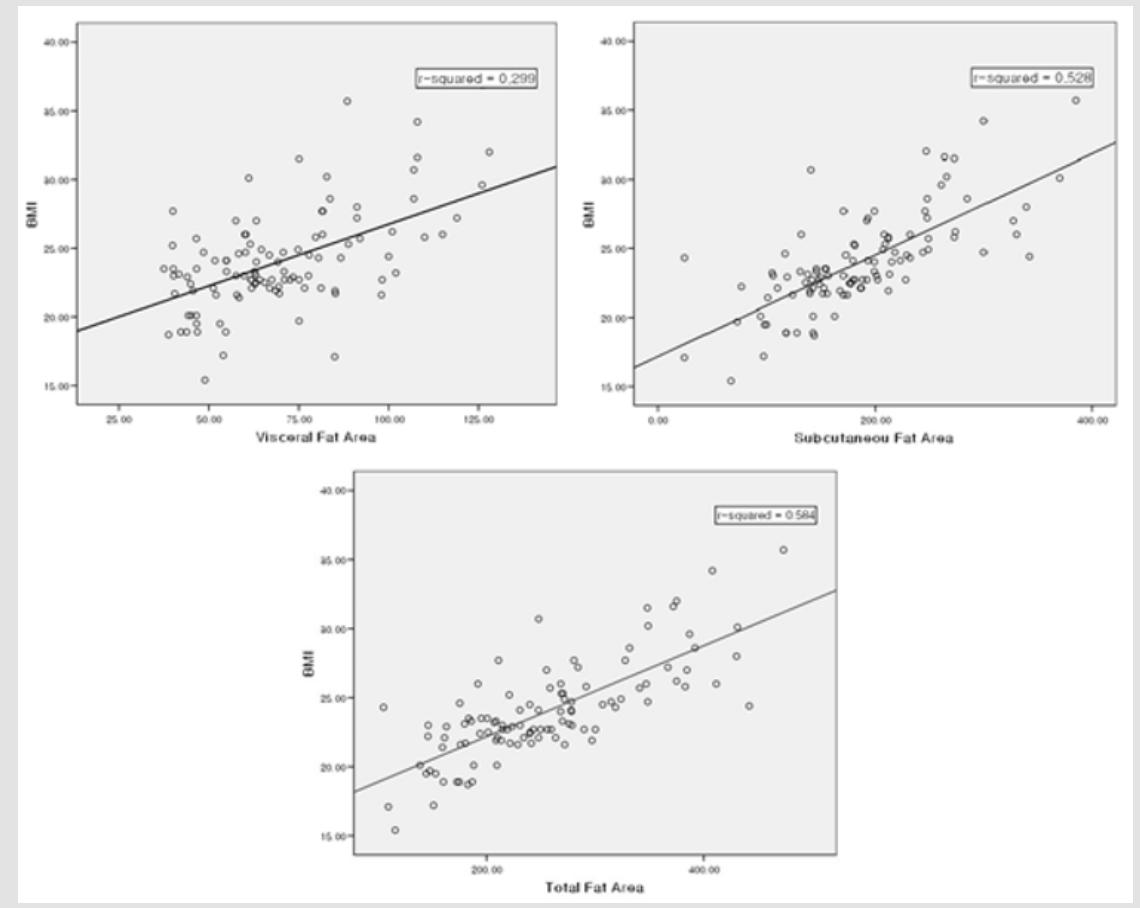

Figure 3: Correlation analyzing BMI among and Fat area.

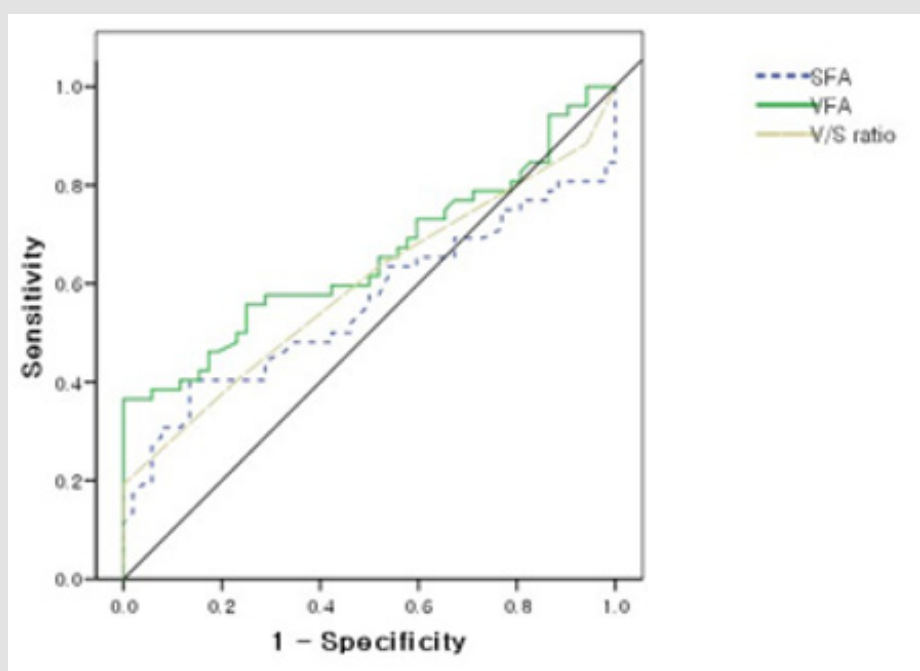

Figure 4: Receiver operating characteristic curves for VFA, SFA, V/S ratio to predict the presence of endometrial cancer.

\section{Adiposity Measurement}

The volume of subcutaneous adipose tissue and visceral adipose tissue was quantified by computed tomography scans (Sensation 64, Siemens Medical Solutions, Forchheim, Germany) with a $16 \mathrm{~mm}$ $\times 0.75 \mathrm{~mm}$ collimation, a $420 \mathrm{~ms}$ rotation time, and a tube voltage at $120 \mathrm{kV}$. All CT scans were performed in a supine position. Ten slices $(1 \mathrm{~cm}$ thick) of the abdomen between the fourth and fifth lumbar vertebrae (L4 - L5) were obtained from each patient. Two contours, 
the body perimeter, and the inner margin of the abdominal muscles, were identified using a dedicated workstation (Aquarius 3D Workstation, Tera Recon, San Mateo, CA).

\section{Statistical Analysis}

Quantitative variables were compared using the MannWhitney test, but the results are presented as mean \pm SD. Spearman Correlation Analysis was used to identify the relationship between BMI and 3 other variables (visceral fat area, subcutaneous fat area, and total fat area). To evaluate the accuracy of the 3 measures, Receiver Operating Characteristics (ROC) analysis was performed. The area under the curve (AUC) was computed and the Youden's index is used to detect the optimal cutoff point. The p-values < 0.05 were considered to be statistically significant. All statistical analyses were performed using SPSS version 14.0 (SPSS Inc., Chicago, IL) and R (version 3.1.2).

\section{Result}

\section{Patient Characteristics}

Of the 124 patients who were pathologically diagnosed with endometrial cancer, 66 patients underwent primary surgery. Of these 124 patients, 12 patients treated with the recurrent disease diagnosed in other hospitals, 39 patients' unavailable CT images and 7 patients transferred were excluded. We also ruled out 14 patients because of incomplete medical records. As a result, a total of 52 patients were enrolled to participate in this study. The characteristics of endometrial cancer and a matched group of healthy individuals. Because the groups were matched by age, and BMI, there were no significant differences between the groups. Though there is no significant difference in subcutaneous fat area (SFA) and total fat area (TFA), visceral fat area (VFA) is noticeably elevated in endometrial cancer cases.

\section{Correlation Analyses Among BMI and Fat Area}

To evaluate the correlation power among BMI and Fat area, BMI showed a positive correlation with visceral fat $\left(\mathrm{VF}, \mathrm{r}^{2}=0.299, \mathrm{p}<\right.$ $0.001)$, subcutaneous fat (SF, $\left.r^{2}=0.528, p<0.001\right)$, total fat $\left(\mathrm{TF}, \mathrm{r}^{2}=\right.$ $0.584, p<0.001)$. VFA has the lowest $r^{2}$ which means that visceral fat is the most independent factor from BMI (Fig. 3). This is reflected in the equation, $\mathrm{BMI}=15.219+0.045 \times \mathrm{VF}+0.30 \times \mathrm{SF}$. As TF and SF have a close relationship and multicollinearity between them, they were automatically removed during the statistical analysis.

\section{Cut off Values of the Visceral Fat Area for Discriminating the Subjects with Endometrial Cancer}

A ROC curve for the VFA, SFA and V/S ratio (visceral fat/ subcutaneous fat ratio) which were used to identify subjects with endometrial cancer was created. The AUC reached 0.654 for VFA ( $p$ $=0.007), 0.557(\mathrm{p}=0.315)$ for SFA, 0.594 for $\mathrm{V} / \mathrm{S}$ ratio $(\mathrm{p}=0.097)$ (Fig. 4). With the help of the ROC curve analysis, we managed to establish the cutoff point for the VFA value. At the value of $70.8 \mathrm{~cm} 2$, sensitivity and specificity in the success group reached $55.8 \%$ and $75 \%$, respectively.

\section{Discussion}

There are many studies analyzing the impact of BMI on the risk and prognosis of endometrial cancer. Several papers evaluated the correlation between body fat distribution and endometrial cancer using waist circumference and waist: hip ratio, many have concluded that upper-body fat deposition increases the risk of endometrial cancer [10-12].However, anthropometric data including BMI cannot accurately determine fat distribution. In this study, it is noteworthy that this study used an optimal technique to estimate fat area by CT scan rather than body fat measurement [13]. The study also confirmed that, unlike subcutaneous fat, visceral fat is more directly related to the development of endometrial cancer. Furthermore, this study is the first to suggest that the VFA cutoff point can be used as a parameter for risk of endometrial cancer.

There are several limitations to our study. The risk factors for endometrial cancer are age, diverse hormonal factors such as hormone replacement therapy after menopause, history of oral contraceptives or tamoxifen usage, obesity, family history of cancer, and diabetes. Nevertheless, our study lacks a risk factor assessment due to insufficient clinical data. This bias can tend to overestimate the impact of VFA in patients with endometrial cancer. One other limitation of this study was that our analysis of this cross- sectional data could not provide causal explanations. Further studies are needed to prospectively relate the accumulation of visceral fat to the presence of risk factors. Endometrial cancer patients had a significantly high VFA compared to the control group. On the other hand, there were no differences in SFA and TFA between the two groups As a result, this means that among people of the same height and weight, those with high levels of visceral fat increase the risk of endometrial cancer. These results are supported by recent studies that correlate metabolic active play of visceral fat in carcinogenesis [14]. Interestingly, our study found that TFA did not differ between endometrial cancer patients and controls. This may be due to the high absolute value of the SFA, which is primarily composed of TFAs. In the cancer group, SFA was $194.2 \pm 86.5 \mathrm{~cm}^{2}$, VFA level was $76.2 \pm 25.0 \mathrm{~cm}^{2}$. The strength of the present study is cohort research of well-matched controls for age and BMI. Using BMI- matched controls, we found that visceral fat is not a bystander but independent of BMI. VFA showed the best accurate risk correlation to endometrial cancer than BMI, SFA, and TFA through a correlational and multiple regression analysis. Therefore, although the same BMI patients with increased visceral fat can conclude the higher risk of endometrial cancer.

In breast cancer cases, a notable correlation has been reported between changes in adipocytokine levels and increased breast cancer risk in postmenopausal women [15-17]. As hormone-related cancer, endometrial cancer is thought to have an etiology similar to breast cancer. Likewise, in postmenopausal women, fat cells 
suppressed by endocrine organs, especially visceral fat cells, can be assumed to play an important role in endometrial carcinogenesis.

a) Premenopausal women produce estradiol (E2) in the ovary, but in menopaused women, androgen is produced in adipose tissue converted to estriol (E3) aromatization.

b) Although progesterone has an anti-cancer effect, postmenopausal women have reduced progesterone levels [18].

c) Early postmenopausal is associated with a preferential visceral fat increase regardless of age or total adiposity [19]. These three theories can support our hypothesis.

In this study, we found that VFA was superior to SFA and in V/S ratio in identifying endometrial cancer, as indicated by VFA's larger AUC. Our study also showed that the cutoff points of $70.8 \mathrm{~cm}^{2}$ VFA were optimal in yielding maximal sensitivity and specificity in the prediction of endometrial cancer, using the ROC curve. In similar studies to this one, Hayashi and Miyawaki have set a VFA cut-off point of central obesity in the metabolic syndrome. In the Japanese American study, the VFA cutoff point was set at $75 \mathrm{~cm}^{2}$ [20], and in the Japanese study, the cutoff point set at $65 \mathrm{~cm}^{2}$ [21].

Our research set the cutoff point in between the cutoff points of these studies as it was deemed acceptable to set the cutoff point at a level that increases the risk of central obesity-related disease $[22,23]$. There is a difference in the cutoff points of central obesity according to ethnicity. Since only Korean were analyzed in this study, large-scale research including other ethnicities are needed to produce a reliable cutoff point of central obesity. Furthermore, the addition of a large-scale study to derive a cutoff value that increases the risk of endometrial cancer will increase the awareness of the risk of endometrial cancer in obese women and may help to reduce the incidence.

\section{Conclusion}

This study shows that VFA can be an independent risk factor of endometrial cancer superior to BMI. These results demonstrate the limitations of determining obesity-related cancer risks only through BMI. Furthermore, this study shows the significant impact of visceral fat on the development of endometrial cancer. The study highlights the need for further research regarding the potential physiological and pathological pathways of visceral fat.

\section{Conflict of Interest}

The authors declared no potential conflicts of interest with respect to the research, authorship, and publication of this article.

\section{References}

1. Fontaine KR, Redden DT, C Wang C, Westfall AO, Allison DB (2003) Years of life lost due to obesity. Jama 289(2): 187-193.

2. Ballard-Barbash, R, Berrigan D, Potischman N, Dowling E (2010) Cancer and Energy Balance, Epidemiology and Overview, Springer New York: New York, NY: 1-44.
3. De Pergola G, Silvestris F (2013) Obesity as a Major Risk Factor for Cancer. Journal of Obesity 2013: 11

4. Siegel RL, Miller KD, Jemal A (2016) Cancer statistics, 2016. CA: A Cancer Journal for Clinicians 66(1): 7-30.

5. Oh CM, Won YJ,Jung KW, Kong HJ, Cho H, et al. (2016) Cancer Statistics in Korea: Incidence, Mortality, Survival, and Prevalence in 2013. Cancer research and treatment: official journal of Korean Cancer Association 48(2): 436

6. Reeves GK, Pirie K, Beral V, Green J, Spencer E, et al. (2007) Cancer incidence and mortality in relation to body mass index in the Million Women Study: cohort study. BMJ 335(7630): 1134

7. Potischman N, Hoover RN, Brinton LA, Siiteri P, Dorgan JF, et al. (1996) Case-control study of endogenous steroid hormones and endometrial cancer. Journal of the National Cancer Institute 88(16): 1127-1135.

8. Doyle SL, Donohoe CL, Lysaght J, Reynolds JV (2012) Visceral obesity, metabolic syndrome, insulin resistance and cancer. Proceedings of the Nutrition Society 71(01): 181-189.

9. D'Agostino RB (1998) Propensity score methods for bias reduction in the comparison of a treatment to a non-randomized control group. Stat Med 17(19): 2265-2281.

10. Xu WH, Matthews CE, Xiang YB, Zheng W, Ruan ZX, et al. (2005) Effect of adiposity and fat distribution on endometrial cancer risk in Shanghai women. American journal of epidemiology 161(10): 939-947.

11. Sponholtz TR, Palmer JR, Rosenberg L, Hatch EE, Adams-Campbell LL, et al. (2016) Body Size, Metabolic Factors, and Risk of Endometrial Cancer in Black Women. American journal of epidemiology 183(4): 259-268.

12. Schapira DV, Kumar NB, Lyman GH, Cavanagh D, Roberts WS, et al. (1991) Upper-body fat distribution and endometrial cancer risk. Jama. 266(13): 1808-1811.

13. Yoshizumi T, Nakamura T,Yamane M, Waliul Islam AHM, Menju M, et al. (1999) Abdominal fat: Standardized technique for measurement at ct 1. Radiology 211(1): 283-286.

14. Donohoe CL, Doyle SL, Reynolds JV (2011) Visceral adiposity, insulin resistance and cancer risk. Diabetology \& metabolic syndrome 3: 12 .

15. Macciò A, Madeddu C, Gramignano G, Mulas C, Floris C, et al. (2010) Correlation of body mass index and leptin with tumor size and stage of disease in hormone-dependent postmenopausal breast cancer: preliminary results and therapeutic implications. Journal of molecular medicine 88(7): 677-686.

16. Rose D, Komninou D, Stephenson G (2004) Obesity, adipocytokines, and insulin resistance in breast cancer. Obesity reviews 5(3): 153-165.

17. Mantzoros C, Petridou E, Dessypris N, Chavelas C, Dalamaga M, et al. (2004) Adiponectin and breast cancer risk. The Journal of Clinical Endocrinology \& Metabolism 89(3): 1102-1107.

18. Missmer SA, Eliassen AH, Barbieri RL, Hankinson SE (2004) Endogenous estrogen, androgen, and progesterone concentrations and breast cancer risk among postmenopausal women. Journal of the National Cancer Institute 96(24): 1856-1865

19. Toth MJ, Tchernof A, Sites CK, Poehlman ET (2000) Menopause-related changes in body fat distribution. Annals of the New York Academy of Sciences 904(1): 502-506.

20. Hayashi T, Boyko EJ, McNeely MJ, Leonetti DL, Kahn SE, et al. (2007) Minimum waist and visceral fat values for identifying Japanese Americans at risk for the metabolic syndrome. Diabetes Care 30(1): 120-127.

21. Miyawaki T, Hirata M, Moriyama K, Sasaki Y, Aono H, et al. (2005) Metabolic syndrome in Japanese diagnosed with visceral fat measurement by computed tomography. Proceedings of the Japan Academy, Series B 81(10): 471-479. 
22. Anuurad E, Shiwaku K, Nogi A, Kitajima K, Enkhmaa B, et al. (2003) The new BMI criteria for Asians by the regional office for the western pacific region of WHO are suitable for screening of overweight to prevent metabolic syndrome in elder Japanese workers. Journal of occupational

ISSN: $2574-1241$

DOI: 10.26717/BJSTR.2020.28.004609

Jeong Sig Kim. Biomed J Sci \& Tech Res

(c) (i) This work is licensed under Creative

Submission Link: https://biomedres.us/submit-manuscript.php health 45(6): 335-343.

23. Alberti KGMM, Zimmet P, Shaw J (2006) Metabolic syndrome-a new world-wide definition. A consensus statement from the international diabetes federation. Diabetic medicine 23(5): 469-480.

$\begin{array}{ll}\text { BIOMEDICAL } & \text { Assets of Publishing with us } \\ \text { RESEARCHES } & \text { - Global archiving of articles } \\ \text { - Immediate, unrestricted online access } & \text { - Rigorous Peer Review Process } \\ & \text { - Authors Retain Copyrights } \\ & \end{array}$

